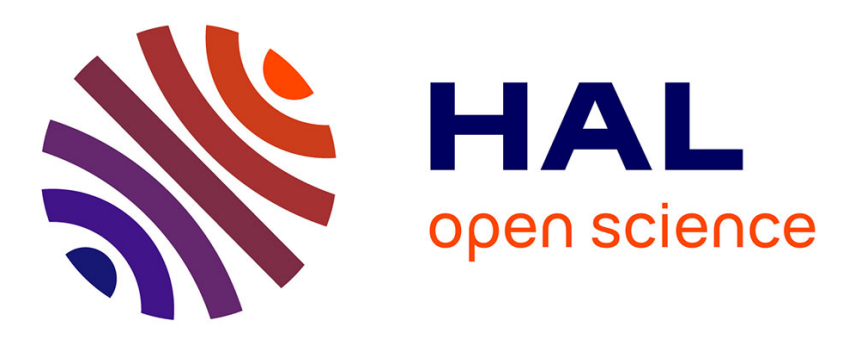

\title{
A general equivalent circuit model for Phoenix cells
}

\author{
A. Guarriello, G. Courtin, Renaud Loison, R. Gillard
}

\section{To cite this version:}

A. Guarriello, G. Courtin, Renaud Loison, R. Gillard. A general equivalent circuit model for Phoenix cells. IEEE Transactions on Antennas and Propagation, 2021, 10.1109/TAP.2021.3076676 . hal03268843

\section{HAL Id: hal-03268843 \\ https://hal.science/hal-03268843}

Submitted on 30 Jun 2021

HAL is a multi-disciplinary open access archive for the deposit and dissemination of scientific research documents, whether they are published or not. The documents may come from teaching and research institutions in France or abroad, or from public or private research centers.
L'archive ouverte pluridisciplinaire HAL, est destinée au dépôt et à la diffusion de documents scientifiques de niveau recherche, publiés ou non, émanant des établissements d'enseignement et de recherche français ou étrangers, des laboratoires publics ou privés. 


\title{
A general equivalent circuit model for Phoenix cells
}

\author{
Andrea Guarriello, Guillaume Courtin, Renaud Loison, Raphael Gillard
}

\begin{abstract}
This communication proposes a general equivalent circuit model for the fast characterization of $N^{t h}$-order Phoenix cells. It provides an intuitive and comprehensive quasi-analytic description of the Phoenix cells' electrical behavior through static lumped reactive elements representation. Given the quasi-analytic nature of the model, it can be exploited in a pre-dimensioning phase. The model proves to give good qualitative accordance with respect to full-wave simulations; statistical analysis on a large set of geometries demonstrates the model efficiency and generality.
\end{abstract}

Index Terms-High-Order Phoenix Cells, Equivalent circuit model, Square loops periodic structures.

\section{INTRODUCTION}

T HE Phoenix cell (PC) was originally presented in [1] as a single layer periodic structure characterized by square loops (Fig. 1). Thanks to its rebirth property, it has the unique capability to come back to its initial geometry after a complete $360^{\circ}$ cycle, thus naturally preventing any abrupt variation in the design of quasi-periodic structures, for instance. In [2] first and second-order PCs are mapped on a sphere surface, highlighting other good PC features.

Figure 1 shows the PC geometries that are investigated in this communication. The order $N$ of the PC is related to the number of independent geometrical parameters defining the concentric square patches and apertures and summarized as $\mathbf{d}=\left[\begin{array}{llll}d_{0} & d_{1} & \ldots & d_{N}\end{array}\right]$. The cell can be further classified by its inductive (periodic distribution of holes in a metallic sheet) or capacitive (periodic distribution of patches) nature.

When single layer structures are required, higher-order PC can be exploited to reduce the substrate thickness while maintaining low dielectric permittivity [3], for enhancing the bandwidth to work with multiple frequency bands and coverages [4]. Higher-order PCs indeed provide more flexibility in the RF response associated with the higher degrees of geometrical freedom.

The scope of this communication is to present a generalized quasi-analytic tool based on lumped circuital elements that allow a qualitative characterization of the scattering behavior of $N^{t h}$-order PCs in a periodic environment with a minimal computational cost. Given the quasi-analytical nature of the proposed model, it can be exploited in large variable optimization problems [5], and in the pre-dimensioning design

This work was supported in part by the French Space Agency (CNES) and Thales Alenia Space France and in part by the European Commission under the H2020 Marie Sklodowska-Curie project REVOLVE, in the frame of two $\mathrm{PhD}$ grants.

A. Guarriello, G. Courtin, R. Loison and R. Gillard are with the Institut d'Electronique et des Technologies du numéRique (IETR), National Institute of Applied Sciences (INSA), 35708 Rennes Cedex 7, France (e-mail: andrea.guarriello@insa-rennes.fr; guillaume.courtin@insa-rennes.fr; renaud.loison@insa-rennes.fr; raphael.gillard@insa-rennes.fr).

A. Guarriello is with the Institute of Sensors Signals and Systems, HeriotWatt University, Edinburgh EH14 4AS, U.K.
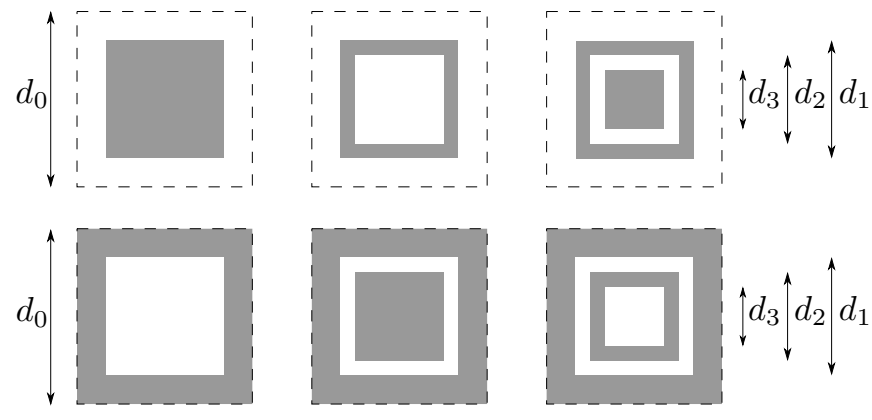

Fig. 1: From left to right Phoenix cells of first, second, and third-order, capacitive type on the top, inductive type on the bottom. The figure represents the cells' metal regions in grey.

phase of complex structures [6]. Moreover, the proposed circuital representations offer intuitive physical insight into the electric behavior of these periodic structures. The circuital elements values are determined through a physically justified combination of pre-existing analytic equations describing the impedance of continuous metal strips [7].

PCs belong to the large family of periodic structures called Frequency Selective Surface (FSS). FSS modeling has seen vast developments since the early '50 [7].

Different techniques enable the analysis and design of such structures, from which we can distinguish numerical and analytic techniques. The first class, based on full-wave analysis and the Floquet expansion of the scattered field [8], presents the advantage to be general and accurate and the disadvantage to be computationally expensive.

The model presented in this communication belongs to the class of quasi-analytical equivalent circuit approaches. The interaction of a plane wave with an infinite periodic array is modeled through a wave traveling in a transmission line including a lumped impedance representing the grid pattern of the cell [9]. These models take advantage of the combination of reactive lumped circuital elements, such as capacitors and inductors, representing simple periodic dichroic structures [7], or more complex patterns, such as Jerusalem cross elements [10], single [11] or double metal ring elements [12], [13].

Other analytical approaches are based on averaged boundary conditions, as in [14] and [15]. These modeling techniques offer the analytic expressions of the lumped impedance associated with continuous TE-TM polarized metallic strips or metallic patches at oblique incidences. They give an accurate description of the equivalent surface impedance but their field of application is limited to simple structures, such as square patches and apertures (here classified as first-order PCs) and

\section{ACCEPTED MANUSCRIPT}


continuous metal strips.

In this communication, we propose a systematic and recurrent procedure for constructing generalized PCs equivalent circuits under normal incidence conditions. It can be seen as an extension to those available in the literature for low order PCs (first-order capacitive and inductive PC [15], second-order capacitive PCs under normal incidence [11], under oblique incidence [13], fourth-order PCs under normal incidence [12], and oblique incidence [13]). The proposed recurrent procedure allows the extension of the equivalent circuit modeling of simple structures to higher-order PCs while giving a physical insight into the cell behaviour. Indeed the proposed formulation considers the effects of the reactance and susceptance linked to the most internal patch or aperture and it introduces physically justified scaling factors, enabling a general construction of the equivalent circuit model for PCs of order $N$. An exhaustive validation with respect to full-wave simulations is performed to assess the accuracy expected from the use of this model. This extensive validation of equivalent circuit models is a novelty to our best knowledge.

The communication is structured as follows: section II-A presents the model's physical insight. Section II-B explains the criteria adopted for the model construction. In section II-C and II-D, the quasi-analytic formulation is presented. Validation results are presented in section III.

\section{PROPOSED MODEL}

\section{A. Physical analysis of the $P C$}

To have a physical insight on the PC electrical behavior, we consider as an example fourth-order PCs as depicted in Figure 2. The lumped circuital elements associated with the geometrical patterns are issued from the cell response to a $x$ polarized wave.

As a general principle, the metal zones are associated with inductive effects caused by the current charges flow in the metal paths parallel to the electric field. On the other hand, the capacitive reactances are representative of the charges accumulation on the metal edges orthogonal to the electric field. The reactive lumped elements shown in the figure are linked to the geometrical parameters defining the metalized or slotted square loops. For instance, the most external reactive elements (capacitance on the left-sided cell and inductance in the rightsided cell) are referred to as the capacitive susceptance and the inductive reactance of the external grid in the capacitive and inductive cells.

\section{B. General equivalent capacitive cell impedance construction}

This section considers the recurrent construction of the equivalent impedance associated with a capacitive PC of order $N$. The complementary inductive cell impedance can be easily obtained in virtue of the Babinet principle. Figure 3 shows a capacitive PC of order $N$ that can be an odd and even integer. $d_{0}$ is the cell lattice size, while $d_{N}$ is the size of the smallest internal patch (even order) or aperture (odd order). We assume $\lambda \gg d_{0}$, where $\lambda$ is the free-space wavelength.

The generalized physical interpretation makes the recurrent impedance construction of the PC of even and odd order

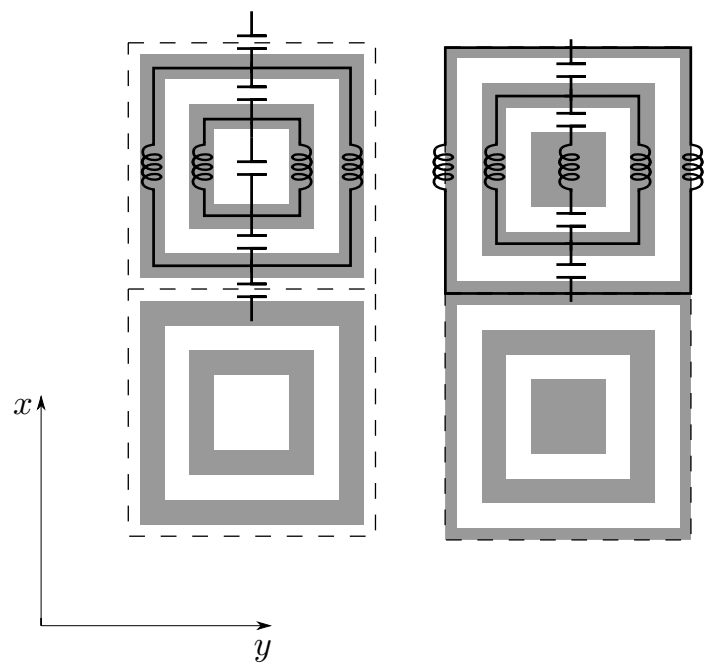

Fig. 2: Representation of the fourth-order Phoenix cells and the associated lumped circuital elements. Two consecutive capacitive cells on the left, two consecutive inductive cells on the right.

straightforward. The equivalent resonator is shown in Figure 4 , in which the equivalent impedance is represented for an even order (a) and an odd order (b). The convention of the reactance's subscripts $X$ and the susceptance $B$ can be easily linked to the geometrical parameters depicted in Figure 3.

By specifying more in detail, the capacitive susceptance $B_{1}$ is associated with the charge accumulation on the metal edges of length $d_{1}$ of two consecutive cells, which are orthogonal to the electric field. The inductive reactance $X_{1,2}$ is related to the charge flow in the symmetric metal paths defined by the lengths $d_{1}$ and $d_{2}$, and it takes into account the series connection of the two symmetric inductances (Fig. 2). This process is applied for the other lumped reactances and susceptances until the circuital element with subscript $N$, which is related to the most internal patch $\left(X_{N}\right.$ associated to the even-order cell), or the most internal aperture ( $B_{N}$ associated with the odd-order cell). The next section introduces general quasi-analytical reactance and susceptance expressions derived from the inductance and strip gratings' capacitances.

\section{Lumped elements characterization from strip gratings for- mulation}

The analytical procedures for deriving the lumped elements values are based on pre-existing analytic equations [7] describing the reactive impedance of continuous metal strips. The same methodology was applied in references [10], [11], [12], [13], where the analytic expressions of strips grating impedance supplied by [7] were introduced.

The response of continuous metallic strips with a 1D periodicity depends on the wave polarization with respect to the axis of periodicity. When the incident electric field is parallel to the strips, the response is inductive, as shown in Figure 5.

From [7] we can express the inductive reactance as a function of the geometrical parameters of the strips and

\section{ACCEPTED MANUSCRIPT}




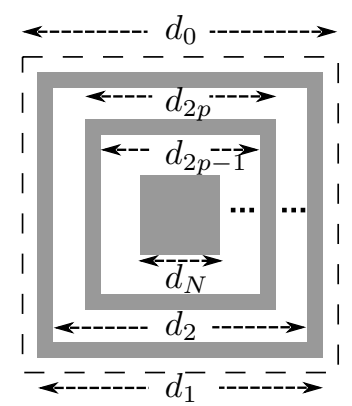

(a)

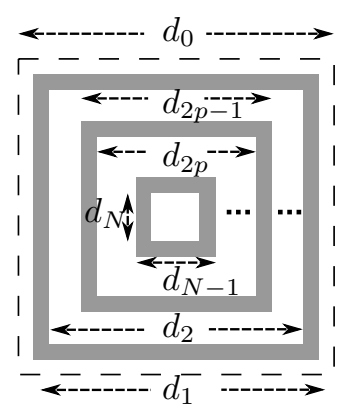

(b)
Fig. 3: Generalized capacitive Phoenix cell geometry of even order (a), and of odd order (b).

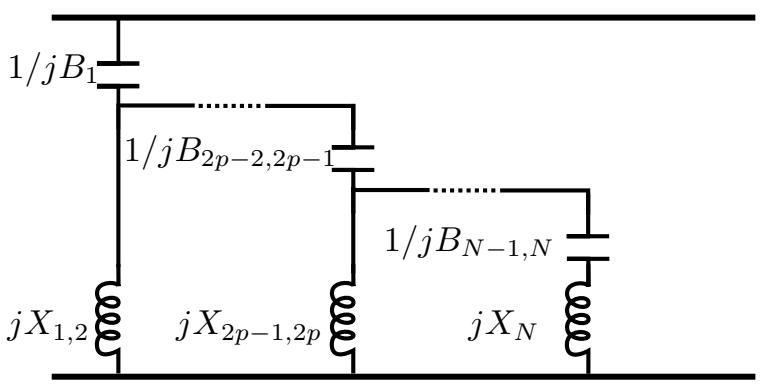

(a)

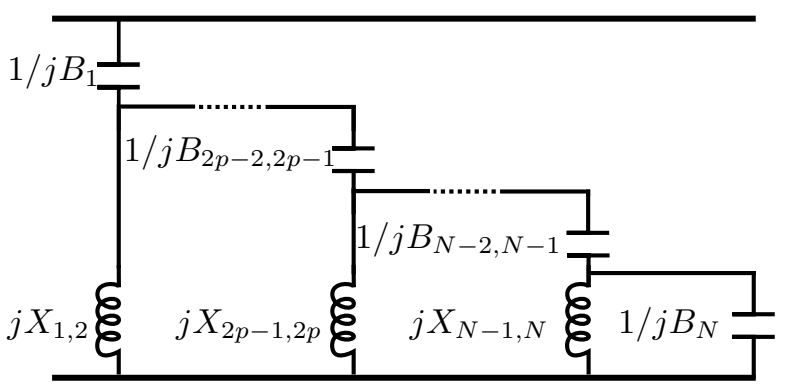

(b)

Fig. 4: Generalized equivalent circuit model of the capacitive Phoenix cell geometry of even order (a), and odd order (b).

the wavelength $\lambda$, where $w$ is the width of the strips and $g=d_{0}-w$ the gap, as shown in Figure 5. The reactance is given by

$$
\begin{aligned}
\frac{X\left(d_{0}, w, \lambda\right)}{\eta_{0}} & =f\left(d_{0}, w, \lambda\right) \\
& =\frac{d_{0}}{\lambda}\left[\ln \csc \frac{\pi w}{2 d_{0}}\right]
\end{aligned}
$$

The plane wave free-space impedance $\eta_{0}$ for normal incidence is given by $\eta_{0}=\sqrt{\frac{\mu_{0}}{\epsilon_{0}}}$. Similarly, if the electric field is polarized perpendicular to the strips, the response is capacitive. The strips susceptance can be derived from the Babinet principle as

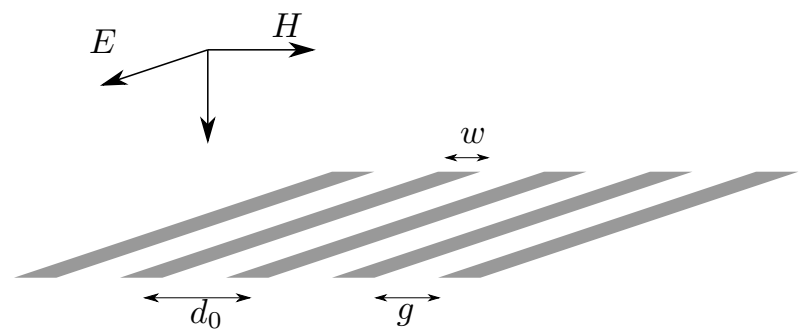

Fig. 5: Periodically organized grid of parallel strips having an inductive response (electric fields parallel to the strips).

$$
\frac{B\left(d_{0}, g, \lambda\right)}{\eta_{0}}=4 f\left(d_{0}, g, \lambda\right) .
$$

For the Phoenix capacitive cell of order $N$, the recurrent expressions describing the susceptances $B$ have the following form

$$
\begin{gathered}
\frac{B_{1}}{\eta_{0}}=4 f\left(d_{0}, d_{0}-d_{1}, \lambda\right) \frac{d_{1}}{d_{0}} \\
\frac{B_{2 p-2,2 p-1}}{\eta_{0}}=\frac{1}{2} 4 f\left(d_{0}, \frac{d_{2 p-2}-d_{2 p-1}}{2}, \lambda\right) \frac{\sqrt{d_{2 p-2} d_{2 p-1}}}{d_{0}} \\
\frac{B_{N}}{\eta_{0}}=4 f\left(d_{0}, d_{N}, \lambda\right) \frac{d_{N}}{d_{0}}
\end{gathered}
$$

while the reactances $X$ are given by

$$
\begin{gathered}
\frac{X_{2 p-1,2 p}}{\eta_{0}}=\frac{1}{2} f\left(d_{0}, \frac{d_{2 p-1}-d_{2 p}}{2}, \lambda\right) \frac{\sqrt{d_{2 p-1} d_{2 p}}}{d_{0}} \\
\frac{X_{N}}{\eta_{0}}=f\left(d_{0}, d_{N}, \lambda\right) \frac{d_{N}}{d_{0}} .
\end{gathered}
$$

The $\frac{1}{2}$ coefficient appearing in equations 4 and 6 respectively takes into account the two symmetric susceptances or reactances that are connected in parallel or series configuration (Fig. 2).

The scaling factors on the right side of equations 3-7 are derived through empirical considerations, as in [10]-[13]. They take into account the finite nature of the strips in the PCs. In our model, the scaling factor is a normalized length representing the average of the geometrical parameters contributing to an element. As an example, we consider the capacitance between two metal strips of different lengths. In this case, the normalized length is the geometric mean of the two strip lengths.

The expression or the value of the lumped elements could certainly be improved. An optimization procedure could be derived in order to find a more accurate value of the impedance as function only of the geometrical parameters. It could be done also by using alternative techniques that can be found in literature, for instance [16], and applied to more complex FSS such as Phoenix cell. However, this is out of the scope of this communication, whose main objective is to provide a computationally fast model. 


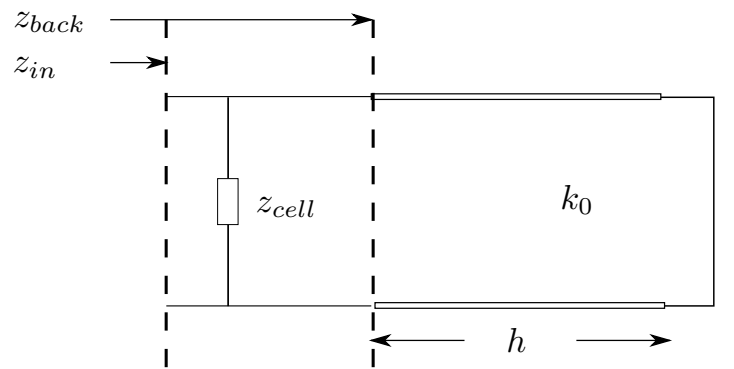

Fig. 6: The transmission-line model for the Phoenix cell on top of a metal-backed air slab.

\section{Final PC model}

The simulation results concern PCs lying on top of a metalbacked air slab. The complete model to compute the reflection coefficient is depicted in Figure 6. The impedance $z_{\text {cell }}$ is derived by applying formulas $3,4,5,6,7$, and by combining the lumped elements as in Figure 4.

The transmission line input impedance $z_{i n}$ is derived from the parallel connection of the cell impedance $z_{\text {cell }}$ and the impedance of the grounded transmission line $z_{\text {back }}=$ $j \tan k_{0} h$, being $k_{0}$ the wave-number in free space and $h$ the height of the air layer. The input impedance is then given by

$$
z_{\text {in }}^{-1}=z_{\text {cell }}^{-1}+z_{\text {back }}^{-1}
$$

from which the derivation of the reflection coefficient $R$ is immediate

$$
R=\frac{z_{\text {in }}-1}{z_{\text {in }}+1} .
$$

The proposed model considers only purely reactive elements. Consequently, the magnitude of the reflection coefficient is assumed to be always $0 \mathrm{~dB}$. This is a realistic assumption as long as sharp resonances are avoided, which is usually a requirement in reflectarray applications. The design only relies on the reflection phase variations, noted in the following as $\phi_{R}$.

It is worth mentioning that the proposed model is intended to provide a very fast and qualitative tool for pre-dimensioning studies of a large set of PC geometries. Indeed, some basic PC design parameters, such as the cells lattice $d_{0}$, the substrate height $h$, the Phoenix cycle parameters (which define the cells smooth geometrical variation versus their reflection properties [1], [2]) can be explored in a preliminary design phase very efficiently [3]. The preliminary design phase is intended to help the designer explore and choose some relevant parameters before a more accurate full-wave analysis, avoiding several computationally expensive EM simulations. The model does not exempt from using a rigorous tool for a final design phase. On the contrary, it allows targeting the accurate characterization of only a few samples after a pre-dimensioning assessment.

\section{Model VAlidation}

The proposed model results are compared to the results of accurate full-wave simulations performed with Ansoft High Frequency Structure Simulator (HFSS).

\section{A. Validation for $4^{\text {th }}$ order PCs}

We consider the $4^{\text {th }}$ order inductive PCs presented in [1] and depicted in Figure 7. The cells are simulated assuming local periodicity in a very wide frequency band $[3-25] \mathrm{GHz}$. The inter-element spacing in the infinite periodic array is set to $d_{0}=12 \mathrm{~mm}$. The cell lies over a ground plane placed at a distance $h=6 \mathrm{~mm}$. The geometrical parameters are reported in table I.

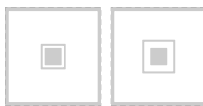

(a) (b)

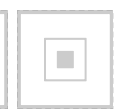

(c)

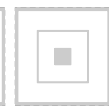

(d)

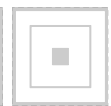

(e)

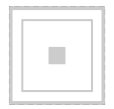

(f)

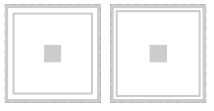

(g) (h)
Fig. 7: Phoenix cycle composed of $4^{\text {th }}$ order inductive cells, as in [1].

TABLE I: Dimensions of $4^{\text {th }}$ order cells.

\begin{tabular}{ccccccccc}
\hline & (a) & (b) & (c) & (d) & (e) & (f) & $(\mathrm{g})$ & $(\mathrm{h})$ \\
\hline$d_{1}(\mathrm{~mm})$ & 11.3 & 11.3 & 11.3 & 11.3 & 11.3 & 11.3 & 11.3 & 11.3 \\
$d_{2}(\mathrm{~mm})$ & 2.6 & 3.8 & 5 & 6.2 & 7.4 & 8.6 & 9.8 & 11 \\
$d_{3}(\mathrm{~mm})$ & 2.3 & 3.5 & 4.7 & 5.9 & 7.1 & 8.3 & 9.5 & 10.7 \\
$d_{4}(\mathrm{~mm})$ & 2 & 2 & 2 & 2 & 2 & 2 & 2 & 2 \\
\hline
\end{tabular}

Figure 8 shows the comparison between the full-wave simulations and the proposed model. The general evolution of the phase of the reflection coefficient is globally well predicted. A shift in the resonance frequencies may be observed, especially for extreme configurations ( $a$ and $h$ ) where very thin slots are involved.

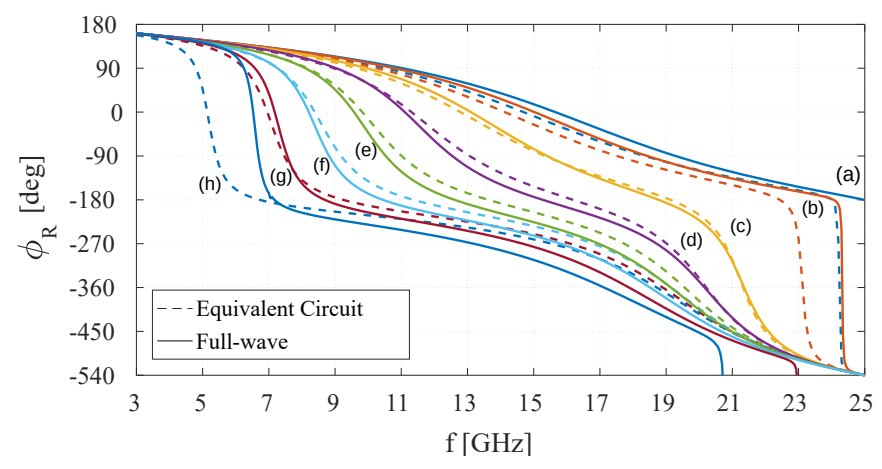

Fig. 8: Reflection phase of $4^{\text {th }}$ order inductive cells.

The results relative to the complementary capacitive cycle of Figure 9, in which the geometrical parameters are kept the same, are shown in Figure 10. For the capacitive cycle, the proposed model's predictions are in good qualitative accordance with the full-wave predictions.

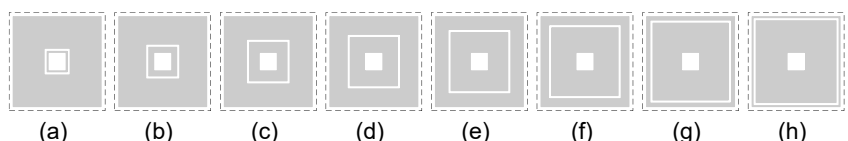

Fig. 9: Phoenix cycle composed of $4^{\text {th }}$ order capacitive cells. 


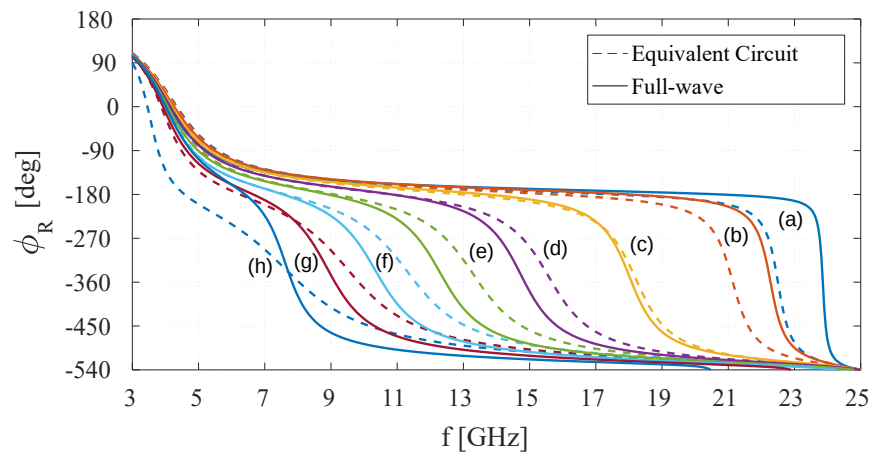

Fig. 10: Reflection phase of $4^{\text {th }}$ order capacitive cells.

For extreme configurations as (a) and (h), the resonance frequencies' prediction is less accurate in both inductive and capacitive cases. The resonance frequencies' shift may be caused by a stronger coupling effect between the thin, long, and close metallic strips. The introduction of a mutual inductance could improve the equivalent circuit as in [17].

\section{B. Statistical analysis}

To provide a complete validation, we now show comparisons for a much larger set of cells. As an example, we consider the reflection phase prediction for 4740 cells forming a database for a dual-band reflectarray [4], where $d_{0}$ is set to $4.9 \mathrm{~mm}$, and $h$ is equal to $2 \mathrm{~mm}$.

Figure 11 presents the complete phase mapping of the cell at $19 \mathrm{GHz}$ and $29 \mathrm{GHz}$ in the $\left[-180^{\circ} ; 180^{\circ}\right]$ range, computed at normal incidence. The map is obtained by varying all the parameters of the cell $\left(d_{1}, d_{2}, d_{3}, d_{4}\right)$ where restrictions are made to avoid overlapping configurations, i.e. $d_{4}<d_{3}<d_{2}<$ $d_{1}$, and to comply with standard fabrication tolerances, i.e. minimum slot width is set to $0.1 \mathrm{~mm}$. The resulting 4740 cells configurations are depicted with blue markers for inductive cells and red markers for capacitive cells. Figure 11 (a) shows the results obtained with full-wave simulations, while Figure 11 (b) shows the results obtained with our equivalent circuit model. The equivalent circuit model can predict the overall behavior of a large set of cells.

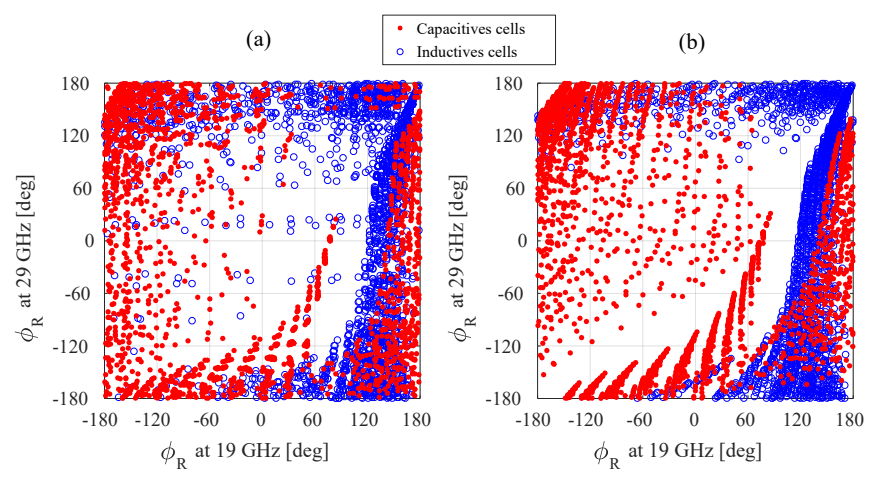

Fig. 11: Databases composed of 4740 cells (a) simulated with HFSS (b) calculated with equivalent circuit model.
We can quantitatively assess the error in predicting the reflected phase from a statistical point of view. Figure 12(a) shows the percentage of cells contained in the database as a function of the error on the reflected phase, at the lower frequency $f_{1}=19 \mathrm{GHz}$. The histograms are differentiated for capacitive cells (in red) and inductive cells (in blue).

For $80 \%$ of the cells, the phase error is less than $45^{\circ}$, which is usually considered as an acceptable deviation regarding cell bandwidth definition [18].

Figure 12(b) shows the same information at the higher frequency $f_{2}=29 \mathrm{GHz}$. We can notice that for $60 \%$ of the cells the error is less than $45^{\circ}$. These plots show that the equivalent circuit model can better predict the cells' behavior at $19 \mathrm{GHz}$ since the $d_{0} \ll \lambda$ hypothesis is more valid for low frequencies. Indeed, in these simulations, we have intentionally used the model out of its recommended domain of validity since $d_{0}$ is almost $\lambda / 2$ at $29 \mathrm{GHz}$. This shows that even in these quite extreme conditions, the prediction is
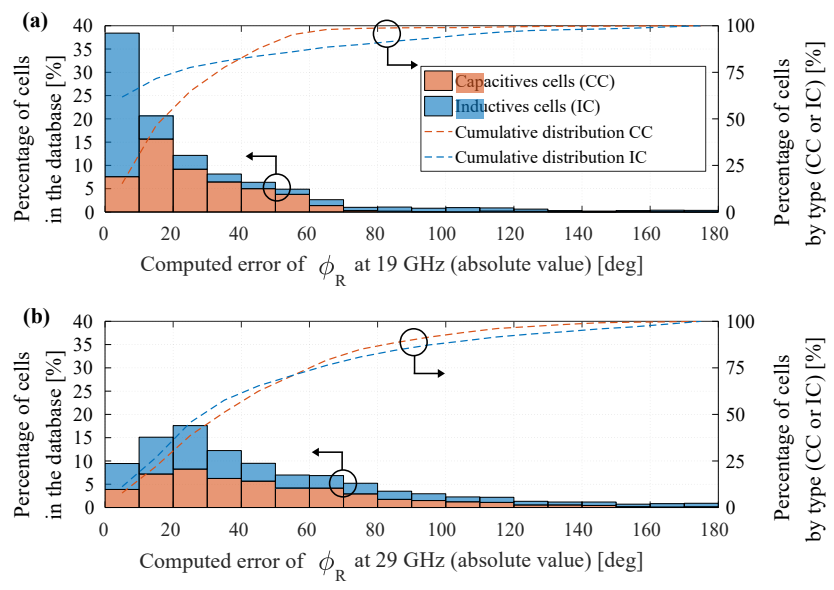

Fig. 12: Distribution of the phase error between the equivalent circuit model and HFSS (a) at $19 \mathrm{GHz}$ and (b) $29 \mathrm{GHz}$.

Finally, to assess the sensitivity of our model with frequency, we also compare the error on frequency dispersion. The normalized frequency dispersion at $f_{i}$ is defined as:

$$
\left|\frac{d \phi_{R}}{d f}\right| \times \frac{f_{i}}{360} \approx\left|\frac{\phi_{R}\left(f_{i}^{+}\right)-\phi_{R}\left(f_{i}^{-}\right)}{f_{i}^{+}-f_{i}^{-}}\right| \times \frac{f_{i}}{360} .
$$

In our simulations $\Delta f_{i}=f_{i}^{+}-f_{i}^{-}$is set to $100 \mathrm{MHz}$. Figures 13(a) and 13(b) show the normalized frequency dispersion error made by our model with respect to full-wave simulations at the two frequencies of interest. Globally, the accuracy of the frequency dispersion is better for capacitive cells. In the worst case, we obtain $80 \%$ of the inductive cells with a frequency dispersion error less than 1 at low frequency and $80 \%$ of the inductive cells with an error less than 1.3 at high frequency. The model remains less accurate at the high frequency, as explained before.

\section{Conclusions}

In this communication, a general equivalent circuit model is proposed for $N^{t h}$-order Phoenix cell (PC) characterization. 

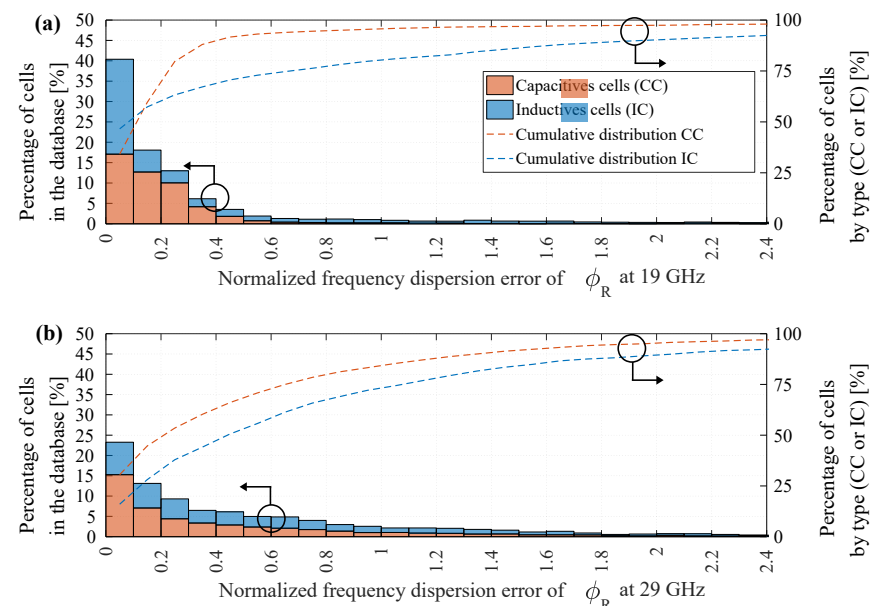

Fig. 13: Distribution of the frequency dispersion error between the equivalent circuit model and HFSS (a) at $19 \mathrm{GHz}$ and (b) $29 \mathrm{GHz}$.

This model allows predicting the reflected phase without any computationally costly full-wave simulation since based on a quasi-analytic formulation. The model proves to give good qualitative accordance with respect to full-wave simulations by predicting fourth-order PCs' frequency evolution behavior with quite a good accuracy even for cells presenting high coupling effects between thin, long, and close metallic strips. A statistical comparison with full-wave simulations shows that for the $80 \%$ of the cells, the phase error is less than $45^{\circ}$ at low frequency and $60 \%$ at a higher frequency. These results demonstrate the proposed equivalent circuit model's capability to be exploited in the preliminary stage of cell design as a fast and simple pre-dimensioning tool before a final design is performed with accurate EM simulations.

\section{REFERENCES}

[1] L. Moustafa, R. Gillard, F. Peris, R. Loison, H. Legay, and E. Girard, "The phoenix cell: A new reflectarray cell with large bandwidth and rebirth capabilities," IEEE Antennas and Wireless Propagation Letters, vol. 10, pp. 71-74, 2011.

[2] V. Richard, R. Loison, R. Gillard, H. Legay, M. Romier, J.-P. Martinaud, D. Bresciani, and F. Delepaux, "Spherical mapping of the second-order phoenix cell for unbounded direct reflectarray copolar optimization," Progress In Electromagnetics Research, vol. 90, pp. 109-124, 2019.

[3] A. Guarriello, R. Loison, G. Goussetis, D. Bresciani, and H. Legay, "Phoenix cells reduced database construction for efficient reflectarray synthesis," in 2019 IEEE International Symposium on Antennas and Propagation and USNC-URSI Radio Science Meeting, 2019, pp. 285286.

[4] G. Courtin, R. Gillard, R. Loison, D. Bresciani, and M. Romier, "Dualband capabilities of the fourth order phoenix cell for reflectarrays antennas," in 2019 International Conference on Electromagnetics in Advanced Applications (ICEAA), 2019, pp. 0647-0650.

[5] A. E. Yilmaz and M. Kuzuoglu, "Design of the square loop frequency selective surfaces with particle swarm optimization via the equivalent circuit model." Radioengineering, vol. 18, no. 2, 2009.

[6] M. Al-Joumayly and N. Behdad, "A new technique for design of low-profile, second-order, bandpass frequency selective surfaces," IEEE Transactions on Antennas and Propagation, vol. 57, no. 2, pp. 452-459, 2009.

[7] N. Marcuvitz, Waveguide handbook. Iet, 1951, no. 21.

[8] M. Guglielmi and A. A. Oliner, "Multimode network description of a planar periodic metal-strip grating at a dielectric interface. i. rigorous network formulations," IEEE Transactions on Microwave Theory and Techniques, vol. 37, no. 3, pp. 534-541, March 1989.
[9] I. Anderson, "On the theory of self-resonant grids," The Bell System Technical Journal, vol. 54, no. 10, pp. 1725-1731, Dec 1975.

[10] R. J. Langley and A. J. Drinkwater, "Improved empirical model for the jerusalem cross," IEE Proceedings $H$ - Microwaves, Optics and Antennas, vol. 129, no. 1, pp. 1-6, February 1982.

[11] R. J. Langley and E. A. Parker, "Equivalent circuit model for arrays of square loops," Electronics Letters, vol. 18, no. 7, pp. 294-296, April 1982.

[12] — "Double-square frequency-selective surfaces and their equivalent circuit," Electronics Letters, vol. 19, no. 17, pp. 675-677, August 1983.

[13] C. K. Lee and R. J. Langley, "Equivalent-circuit models for frequencyselective surfaces at oblique angles of incidence," IEE Proceedings $H$ Microwaves, Antennas and Propagation, vol. 132, no. 6, pp. 395-399, October 1985.

[14] S. Tretyakov, Analytical modeling in applied electromagnetics. Artech House, 2003

[15] O. Luukkonen, C. Simovski, G. Granet, G. Goussetis, D. Lioubtchenko, A. V. Raisanen, and S. A. Tretyakov, "Simple and accurate analytical model of planar grids and high-impedance surfaces comprising metal strips or patches," IEEE Transactions on Antennas and Propagation, vol. 56, no. 6, pp. 1624-1632, June 2008.

[16] S. Maci, M. Caiazzo, A. Cucini, and M. Casaletti, "A pole-zero matching method for ebg surfaces composed of a dipole fss printed on a grounded dielectric slab," IEEE Transactions on Antennas and Propagation, vol. 53, no. 1, pp. 70-81, 2005.

[17] K. Nadaud, R. Gillard, E. Fourn, and H. W. Gundel, "Equivalent circuit of a reconfigurable triple-slot reflectarray cell," IET Microwaves, Antennas Propagation, vol. 10, no. 10, pp. 1080-1086, 2016.

[18] M. Bozzi, S. Germani, and L. Perregrini, "Performance comparison of different element shapes used in printed reflectarrays," IEEE Antennas and Wireless Propagation Letters, vol. 2, pp. 219-222, 2003. 